\title{
New social adaptability index predicts overall mortality
}

Alexander Goldfarb-Rumyantzev ${ }^{1}$, Anna Barenbaum², James Rodrigue ${ }^{3}$, Preeti Rout ${ }^{1}$, Ross Isaacs ${ }^{4}$, Kenneth Mukamal ${ }^{5}$

1Division of Nephrology, Beth Israel Deaconess Medical Center and Harvard Medical School, Boston, USA

2Tel Aviv University, Tel Aviv, Israel

${ }^{3}$ Transplant Institute, Beth Israel Deaconess Medical Center and Harvard Medical School, Boston, USA

${ }^{4}$ Central Virginia Community Health Center, New Canton, USA

${ }^{5}$ Division of General Medicine, Department of Medicine, Beth Israel Deaconess Medical

Center and Harvard Medical School, Boston, USA

Submitted: 2 August 2010

Accepted: 27 August 2010

Arch Med Sci 2011; 7, 4: 720-727

DOI: 10.5114/aoms.2011.24145

Copyright (c) 2011 Termedia \& Banach

\section{Abstract}

Introduction: Definitions of underprivileged status based on race, gender and geographic location are neither sensitive nor specific; instead we proposed and validated a composite index of social adaptability (SAI).

Material and methods: Index of social adaptability was calculated based on employment, education, income, marital status, and substance abuse, each factor contributing from 0 to 3 points. Index of social adaptability was validated in NHANES-3 by association with all-cause and cause-specific mortality.

Results: Weighted analysis of 19,593 subjects demonstrated mean SAI of 8.29 ( $95 \% \mathrm{Cl} 8.17-8.40)$. Index of social adaptability was higher in Whites, followed by Mexican-Americans and then the African-American population (ANOVA, $p<0.001)$. The SAI was higher in subjects living in metropolitan compared to rural areas (T-test, $p<0.001$ ), and was greater in men than in women (T-test, $p<0.001$ ). In Cox models adjusted for age, comorbidity index, BMI, race, sex, geographic location, hemoglobin, serum creatinine, albumin, cholesterol, and glycated hemoglobin levels, SAI was inversely associated with mortality (HR 0.87 per point, $95 \% \mathrm{Cl} 0.84-0.90, p<0.001$ ). This association was confirmed in subgroups.

Conclusions: We proposed and validated an indicator of social adaptability with a strong association with mortality, which can be used to identify underprivileged populations at risk of death.

Key words: outcome, survival, social adaptability, disadvantaged population, disparity, underprivileged.

\section{Introduction}

Disparities in healthcare and clinical outcomes have been documented, and it has been demonstrated that socially disadvantaged individuals may have inferior medical outcomes; in particular, African Americans [1, 2], women [3], and residents of rural as opposed to urban regions [4] are generally considered at risk of inferior outcome. However, defining an underprivileged population based on rigid criteria of skin color, gender, or geographic location may be prone to errors lacking specificity and sensitivity. While some associations between race, gender, geographic
Corresponding author: Alexander S. GoldfarbRumyantzev MD, PhD Division of Nephrology Beth Israel Deaconess Medical Center

185 Pilgrim Rd, FA-832 Boston, MA 02215, USA Phone: 617-632-9880 Fax: 617-667-5276 E-mail: agoldfar@bidmc.harvard.edu 
location and the outcome may be causal, they more likely represent a mix of genetic, socio-economic, and cultural factors. Separating these factors might provide more information about the mechanism of the observed association.

Strong evidence suggests that lower socioeconomic status is a health risk independent of genetic and many clinical factors. For example, we and others have previously described the role of employment status [5], education level [6], insurance status [6], marital status [7, 8], and substance abuse [9] in the clinical outcome. Consistent with this are other reports showing that low education level has a negative association with health status [10]; income has substantial effects on mortality [11]; and Medicaid beneficiaries are less likely to receive optimal treatment and their outcome is worse compared with privately insured patients for common conditions such as myocardial infarction and bronchial asthma [12, 13].

Because definitions of underprivileged status based on race, gender and geographic location are neither sensitive nor specific, we sought to define a combined index of several indicators of socioeconomic status that would more strongly and accurately identify individuals at greatest health risk. To our knowledge, one previous effort to develop such a measure proposed an index based on employment, accommodation and living situation; this index was only weakly associated with symptoms, quality of life, global functioning and disability [14]. We previously proposed a Social Adaptability Index (SAI), a composite indicator based on employment, education level, income, marital status, and substance abuse. This index had a strong and significant association with mortality in patients with chronic kidney disease [15].

In this project, we hypothesized that underprivileged populations experience disparities in outcome at least in part due to lower levels of social adaptability leading to lower education, inferior employment status, lower income, and other social discrepancies. Because definitions of underprivileged status based on race, gender and geographic location are neither sensitive nor specific, we aim to validate a previously proposed SAI, which is a combined index of several indicators of socioeconomic status that would strongly and accurately identify individuals at greatest health risk.

\section{Material and methods}

\section{Source of data}

The study was approved by the Institutional Review Board at the Beth Israel Deaconess Medical Center. We used the data from the Third National Health and Nutrition Examination Survey (NHANES-3) cohort linked to mortality data by a unique ID number. The
NHANES-3 includes a nationally representative sample of the US population when properly weighted for the complex sampling design. Over 30,000 subjects were enrolled in the survey between 1988 and 1994. We excluded individuals younger than 18 years of age from this analysis. The National Center for Health Statistics (NCHS) has linked NHANES-3 survey data with the National Death Index (NDI), to provide information on subject mortality through December 31, 2000.

We first sought to develop and describe an SAI [15] in the general US population and then to determine its association with all-cause mortality, the primary outcome of the study. Records with missing outcome were deleted. Follow-up was censored at death, loss to follow-up, or study completion, whichever occurred first.

\section{Primary variables of interest}

We previously described the calculation of the SAI [15] and used the same approach in this project; we graded each of the individual components on a scale of 0 to 3 (except for income level, which was graded 0 to 2) without additional weighting. This eliminated the problem of scalability, since each individual indicator contributed almost equally to the final index, and we subsequently tested the components individually to ensure they were similarly associated with mortality. In all cases, we used categorizations similar to our previous work $[5,6,8,15]$ to ensure comparability and transparency. The components of the SAI were categorized as follows.

Employment status: $0=$ unemployed, not working due to medical conditions, not working by choice; 1 = retired; 2 = working part time; 3 = working full time.

Education level: 0 = did not complete high school; 1 = high school graduate; 2 = college graduate; 3 = post-college education or doctorate degree.

Marital status: 0 = not married (including never been married and widowed); 1 = divorced or separated; 2 = married without children; 3 = married with children. The married category includes those married with spouse in household, married with spouse not in household, living as married, and living with partner. If a subject was married and the number of people in the household was greater than 2, we considered that individual married with children (marital status $=3$ ), otherwise we considered the individual married without children ( marital status $=2$ ).

Substance abuse: 0 = abusing drugs, alcohol, and tobacco; 1 = abusing two of three substances; 2 = abusing one of three substances; $3=$ none. Alcohol addiction was defined as positive if any of the following criteria were true: (1) number of days 
subjects used more than nine drinks over the last 12 months was greater than 24; (2) number of days subjects used more than five drinks over the last 12 months was greater than 48; (3) number of days subject drank alcohol in past 12 months was greater than 240. Drug addiction was defined based on marijuana or cocaine use history available in the data. In particular, drug addiction was defined as positive if the subject used marijuana or cocaine at least once over the last month. Finally, tobacco addiction was defined as positive if any of the following factors were true: (1) subject described himself/herself as a smoker at the time of interview; (2) subject smoked cigarettes immediately prior to interview (within $30 \mathrm{~min}$ ); (3) number of cigarettes/cigars/pipes/chewing tobacco containers used in the past 5 days was greater than four.

Income: $0=\langle \$ 20 \mathrm{~K} /$ year per household, $1=$ $<$ \$20-50K/year per household; 2 = > \$50 K/year per household. Income categories greater than $\$ 50 \mathrm{~K} /$ year were not available in NHANES-3 for our study population.

Social adaptability index. The SAI was calculated based on the five components described above as previously reported [15]. To calculate SAI, we used a linear combination of factors weighted equally to maximize its implementation and ease of use; as above, this assumption was further tested with mortality.

\section{Covariates}

We used the following covariates in multivariate analysis: age, sex, race, history of diabetes, other comorbid conditions, body mass index, geographic location (urban/rural), hemoglobin concentration, serum creatinine, albumin, cholesterol, and glycated hemoglobin levels.

Race and ethnicity were combined into four groups: non-Hispanic white, non-Hispanic black, Mexican-American, and other. Comorbid conditions were analyzed as a comorbidity coefficient similar to the Charlson comorbidity index [16], where each of the comorbid conditions available in the dataset contributed one point to the composite index with additional points given for older age. Geographic location is defined by NHANES as two categories: $1=$ central or fringe counties of metropolitan areas of 1 million population or more, and $2=$ other.

\section{Statistical analysis}

Since the design of the data collection for NHANES 3 was based on the large oversampling of young children, older persons, black persons, and Mexican Americans, the sampling weights are used in the analysis to appropriately estimate prevalence, means, medians, and other statistics. The sampling weights are provided with the NHANES data. For descriptive statistics and survival analysis we analyzed both weighted and non-weighted data. Since the results of the survival analysis of the weighted and non-weighted data were essentially the same, and because the tool to calculate the C-index was available to us in the SAS environment, but not in the SUDAAN environment, the calculation of the C-index was based only on the non-weighted data.

Continuous variables were summarized using means and standard deviations. Distributions that were skewed or had severe outliers were summarized using the median and interquartile range $\left(25^{\text {th }}, 75^{\text {th }}\right.$ percentiles). Categorical variables were summarized as percent of total. Groups were compared using t-test or ANOVA for continuous variables and $\chi^{2}$ for the categorical variables. Kaplan-Meier plots and Cox proportional hazard models were used for the survival analysis. Survival was defined as the time from the day of the interview to the death or censor. The discrimination ability of the model was estimated using the C-index, described elsewhere [17].

SAS (SAS Institute, Cary, North Carolina) was used for statistical analysis; to assess the effect of complex survey design on the results SUDAAN [18] (Research Triangle Institute, Research Triangle Park, North Carolina) was used for the survival analysis of the weighted data.

\section{Results}

\section{Baseline characteristics}

The study population consisted of 19,593 subjects. Weighted analysis yielded a mean age of 43.8 years ( $95 \% \mathrm{Cl} 43.0-44.6), 47.6 \%$ males, $76.2 \%$ white, $11.1 \%$ African American, and $5.2 \%$ Mexican American. Of the study population, $5.3 \%$ had diabetes and the average number of other comorbid conditions was $2.57(95 \% \mathrm{Cl}$ 2.55-2.59). Other baseline characteristics of the study population, including the distribution of the factors used in calculation of the SAI, are presented in Table I. The SAI was broadly distributed in the study population (Figure 1) with a mean of 8.29 (95\% Cl 8.17-8.40). Of the study population, $17 \%$ died during follow-up. The most frequent causes of death were acute myocardial infarction (10.5\%), other forms of coronary artery disease and atherosclerosis (18.1\%), cerebrovascular disease (8.4\%), malignant diseases of the upper and lower respiratory system (5.7\%) and of the gastrointestinal tract (4.9\%).

\section{The distribution of the SAI in the subgroups}

The distribution of the SAI (mean and 95\% Cl) in subgroups divided by race, gender, and geographic 
Table I. Baseline characteristics of the study population*

\begin{tabular}{|lc|}
\hline Age at interview [years] & $43.8(43.0-44.6)$ \\
\hline Race/ethnicity: & \\
\hline Non-Hispanic white & 76.2 \\
\hline Non-Hispanic black & 11.1 \\
\hline Mexican American & 5.2 \\
\hline Other & 7.5 \\
\hline Sex: & \\
\hline Female & 52.4 \\
\hline Male & 47.6 \\
\hline Body mass index [m/cm²] & $44.0(43.69-44.31)$ \\
\hline Comorbidity index & $2.57(2.55-2.59)$ \\
\hline Diabetes: & 5.3 \\
\hline Yes & 94.7 \\
\hline No & $14.2(14.09-14.21)$ \\
\hline Hemoglobin [g/dl] & $1.07(1.06-1.08)$ \\
\hline Serum creatinine [mg/dl] & $4.19(4.15-4.23)$ \\
\hline Serum albumin [g/dl] & $202.7(202.2-204.2)$ \\
\hline Serum cholesterol [mg/dl] & $5.35(5.30-5.39)$ \\
\hline Glycated hemoglobin [\%] & 49.8 \\
\hline Geographic location: & 25.6 \\
\hline Metropolitan area & \\
\hline Rural area & \\
\hline Education level: & \\
\hline Not completed high school & \\
\hline
\end{tabular}

\begin{tabular}{|c|c|}
\hline High school graduate & 54.6 \\
\hline College graduate & 11.3 \\
\hline $\begin{array}{l}\text { Post-college education } \\
\text { or doctorate degree }\end{array}$ & 8.5 \\
\hline \multicolumn{2}{|l|}{ Marital status: } \\
\hline Not married & 26.3 \\
\hline Divorced or separated & 10.5 \\
\hline Married without children & 24.2 \\
\hline Married with children & 39.0 \\
\hline \multicolumn{2}{|l|}{ Employment status: } \\
\hline Not working (but not retired by age) & 19.6 \\
\hline Retired & 12.1 \\
\hline Working part time & 1.4 \\
\hline Working full time & 66.9 \\
\hline \multicolumn{2}{|l|}{ Addictions to substances: } \\
\hline Tobacco, alcohol, and drugs & 2.4 \\
\hline Two out of three above & 9.8 \\
\hline One out of three above & 28.5 \\
\hline No addictions & 59.3 \\
\hline \multicolumn{2}{|l|}{ Income level: } \\
\hline$<\$ 20 \mathrm{~K} /$ year per household & 35.0 \\
\hline$<\$ 20-50 \mathrm{~K} /$ year per household & 42.3 \\
\hline$>\$ 50 \mathrm{~K} /$ year per household & 22.7 \\
\hline Social adaptability index & $8.29(8.17-8.40)$ \\
\hline
\end{tabular}

*Continuous variables presented as mean (95\% confidence limits of mean), categorical variables presented as percent of total

location is presented in Figure 2. We observed the highest SAl in Whites (8.57 [95\% Cl 8.45-8.69]), followed by Mexican Americans (7.54 [95\% Cl 7.387.70]) and the African American population (7.02 [95\% Cl 6.84-7.20]) (ANOVA, $p<0.001$ ). The SAl was higher in subjects living in metropolitan (8.49 [95\% Cl 8.34-8.65]) compared to rural areas (8.08 [95\% Cl 7.86-8.30]) (T-test, $p<0.001)$ and was greater in men $(8.56[95 \% \mathrm{Cl} 8.45-8.68])$ than women (8.03 [95\% Cl 7.08-8.18]) (T-test, $p<0.001)$.

\section{Survival analysis}

We first evaluated the individual variables comprising SAl in a single multivariate model. All of them except for education level demonstrated significant and reasonably similar associations with survival (Table II).

The association between SAI and mortality was next evaluated in the entire study population and in several subgroups. The SAI was divided into quintiles and Kaplan-Meier plots suggested a graded relationship between the SAI and mortality
(Figure 3), where the mortality increases incrementally with the incremental decrease in SAI.

In multivariable analyses adjusted for age, comorbidity index, BMI, race, sex, geographic location (urban vs. rural), hemoglobin, serum creatinine, serum albumin, serum cholesterol, and glycated hemoglobin levels (Table III), there

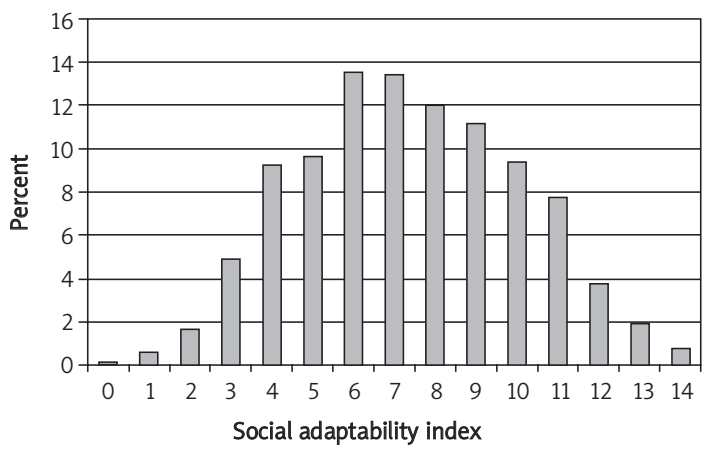

Figure 1. Distribution of SAI values in the study population 

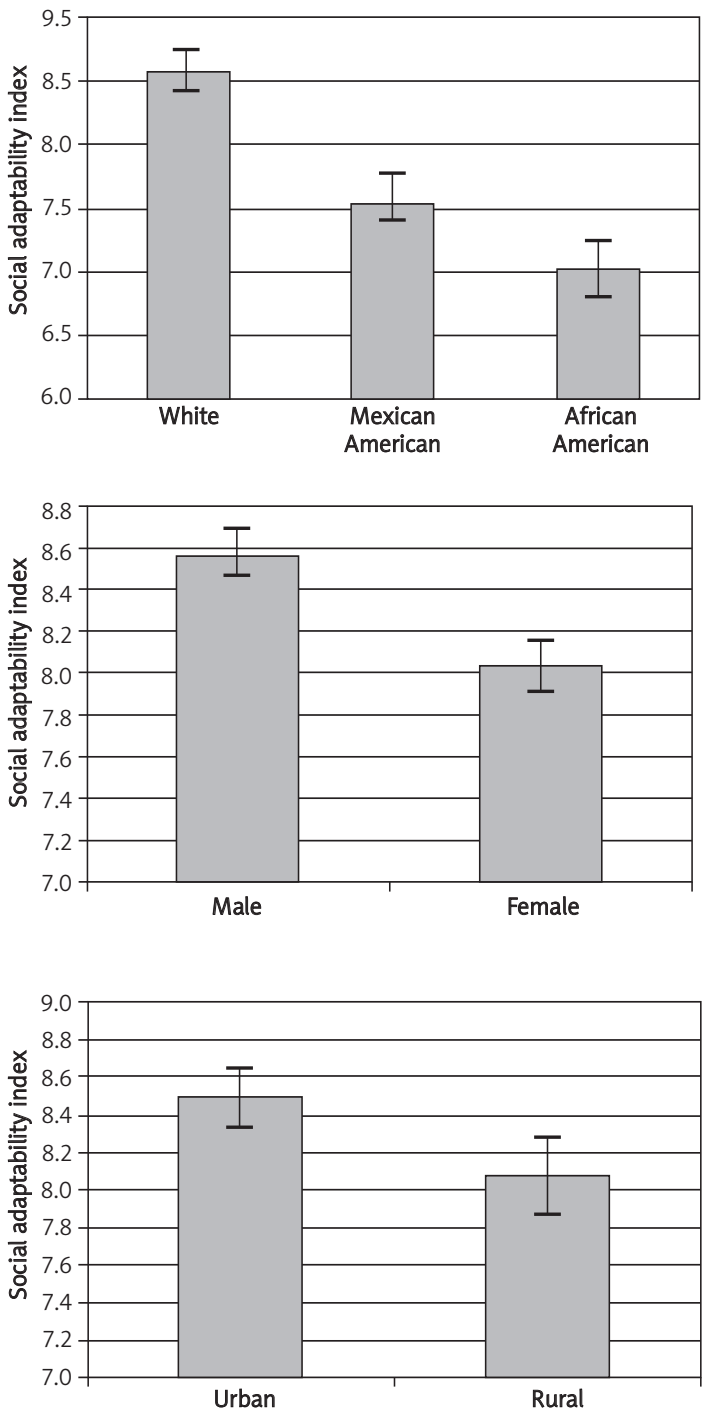

Figure 2. Distribution of SAI (mean and $95 \% \mathrm{Cl}$ ) in subpopulations divided by race, gender, and geographic location

Table II. Results of proportional hazard model of factors used to calculate SAI adjusted for potential confounders*

\begin{tabular}{|lcc|}
\hline & Hazard ratio $(95 \% \mathrm{Cl})$ & Value of $p$ \\
\hline Education level & $0.97(0.91-1.05)$ & 0.54 \\
\hline Marital status & $0.92(0.86-0.98)$ & 0.017 \\
\hline Employment status & $0.89(0.83-0.95)$ & $<0.001$ \\
\hline Substance addiction & $0.76(0.67-0.87)$ & $<0.001$ \\
\hline Income level & $0.75(0.66-0.86)$ & $<0.001$ \\
\hline
\end{tabular}

*In addition to the variables indicated in the table, the model was adjusted for the following covariates: age, sex, race, comorbidity index, history of diabetes, body mass index, geographic location (rural vs. urban), hemoglobin level, serum creatinine level, serum albumin level, serum cholesterol level, and glycated hemoglobin level. All five factors of $S A I$ indicated in the table were graded on the scale from 0 to 3 , except for the income level, which was graded on the scale of 0 to 2, as described in the text continued to be a dose-response relationship between higher quintiles of SAI and lower mortality. Similarly, when analyzed as a continuous variable in the entire study population, SAI had a significant association with survival (HR 0.87, 95\% Cl 0.84-0.90, $p<0.001$ ) (Table IV).

As comorbidity might be a significant confounding factor, our model was adjusted for the Charlson comorbidity index. In additional analysis we included in the model a self-reported indicator of health status as rated by the subjects on the scale of 1 (excellent) to 5 (poor). When this indicator of health status was included in the model, the association of SAI with mortality was unchanged, while the health status indicator itself was found to have a significant association with mortality (HR 1.21, $p<0.001,95 \%$ Cl 1.16-1.26).

Furthermore, we analyzed cause-specific mortality for the three main causes of death: cardiovascular disease (including cerebrovascular disease), malignancy, and diseases of the respiratory system. A separate Cox model was analyzed for each cause of death and those subjects who either survived, or died from other causes, were censored. SAl showed a significant association with all three main causes of death, specifically: death from cardiovascular disease (HR 0.86, $p<0.001$, $95 \% \mathrm{Cl} 0.82-0.90)$, death from malignancy (HR 0.94, $p=0.018,95 \% \mathrm{Cl} 0.89-0.99)$, and death from diseases of the respiratory system (HR 0.83, $p<0.001,95 \% \mathrm{Cl} 0.75-0.91$ ).

To evaluate the discrimination ability of the model we calculated the C-index [17], which can be interpreted as the probability that a subject from the event group has a higher predicted probability of having an event than a subject from the nonevent group.

Removing SAI from the model decreased the C-index from $0.64(95 \% \mathrm{Cl} 0.60-0.67)$ to 0.62 (95\% Cl 0.59-0.66). While the magnitude of change is somewhat modest, it is comparable to other established risk factors. For example, the C-index was essentially unchanged when the comorbidity index, diabetic status, or race was removed from the model; and the $\mathrm{C}$-index decreased to 0.58 (95\% $\mathrm{Cl} 0.55-0.62)$ when age was removed from the model.

In addition we performed subgroup analysis, where groups were defined based on subjects' sex, race, and urban/rural location. The association between SAI and survival was found to be robust and present in every subgroup evaluated in this project (Table IV).

Finally, we analyzed non-weighted data using the same design of the Cox models. The results of this analysis were essentially the same as the results of analysis of the weighted data described above. 


\section{Discussion}

Inferior outcomes in underprivileged populations $[1,3,4,6]$, and the role of socioeconomic characteristics $[19,20]$ have been documented. However, the definition of at-risk groups in these studies is primarily based either on skin color, income, geographic location, gender, or other defined indicators, which might not be either sensitive or specific. We postulated that social maladaptation is more strongly associated with "underprivileged" status in the society than these traditionally defined characteristics. We previously identified the association between education [6], insurance status [6], substance abuse [9], marital status [8], employment [5] and healthcare outcomes. While previous attempts to develop a single integrated index reflecting socioeconomic status have not been very successful [14], we proposed a composite index based on five indicators of socio-economic status (SAI) [15] in patients with chronic kidney disease. In this report we studied the association between SAI and mortality in the general US population. We postulated that this index may be used as an accurate and practical measure of underprivileged status and demonstrated its association with patient survival. For such an indicator to be practical, variables comprising the index should be easily measurable and quantifiable.

Having these factors completely independent is probably not realistic, as they relate to each other to some extent (it has been demonstrated that education is associated with higher income [21], and marital status is associated with higher access to material resources [22]). However, there is a substantial independent component to each of the indicators, as demonstrated in this analysis. In our analysis all but one factor (education level) were independently associated with the primary outcome when analyzed in the same multivariable model. The decision to include education in the SAI calculation was based on the fact that it had a strong and significant association with mortality when analyzed in a separate model, as well as based on the results of our previous studies $[6,15]$.

We demonstrated that SAI does have an association with generally accepted indicators of belonging to an underprivileged population - in particular it tends to be lower in the population defined as "underprivileged" based on race, gender, and rural geographic location. It therefore confirmed the notion that racial minorities, women and rural dwellers have a greater chance to be underprivileged and experience healthcare disparities. Furthermore, the SAI was convincingly validated by survival analysis. Every additional point in SAI reduced the risk of death roughly by $13 \%$. These results are not only statistically significant, but also

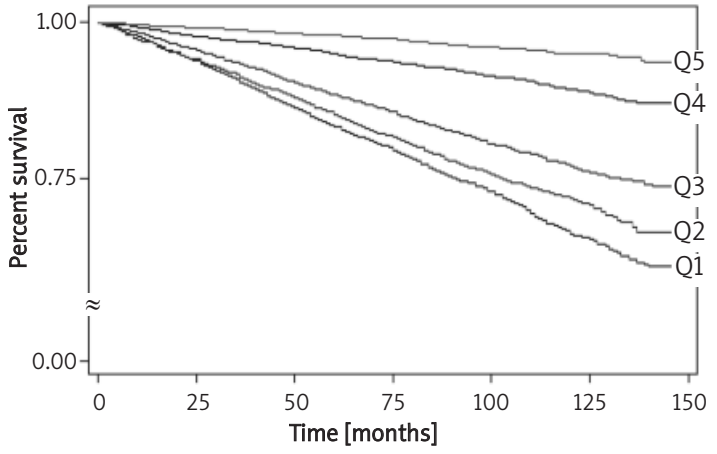

Figure 3. Kaplan-Meier survival curves of the subgroups of subjects divided by the SAI quintile

Table III. The SAI association with mortality in the entire population and study subgroup by proportional hazard model. SAI expressed in categorical format divided into quintiles

\begin{tabular}{|c|c|c|}
\hline Parameters & Hazard ratio $(95 \% \mathrm{Cl})$ & Value of $p$ \\
\hline SAI quintile $1(<5)$ & Reference & \\
\hline SAI quintile 2 (5) & $0.88(0.72-1.07)$ & 0.2 \\
\hline SAl quintile 3 (6-7) & $0.74(0.63-0.87)$ & $<0.001$ \\
\hline SAI quintile 4 (8-9) & $0.58(0.44-0.75)$ & $<0.001$ \\
\hline SAl quintile $5(>9)$ & $0.37(0.27-0.49)$ & $<0.001$ \\
\hline $\begin{array}{l}\text { Age at interview } \\
\text { [years] }\end{array}$ & $1.17(1.13-1.22)$ & $<0.001$ \\
\hline Male sex & Reference & \\
\hline Female sex & $0.48(0.41-0.55)$ & $<0.001$ \\
\hline $\begin{array}{l}\text { Race: non-Hispanic } \\
\text { white }\end{array}$ & Reference & \\
\hline $\begin{array}{l}\text { Race: non-Hispanic } \\
\text { black }\end{array}$ & $0.97(0.80-1.16)$ & 0.71 \\
\hline $\begin{array}{l}\text { Race: Mexican } \\
\text { American }\end{array}$ & $0.995(0.81-1.21)$ & 0.96 \\
\hline Race: other & $0.41(0.23-0.71)$ & 0.002 \\
\hline Comorbidity index & $1.17(1.13-1.22)$ & $<0.001$ \\
\hline $\begin{array}{l}\text { History of diabetes } \\
\text { (compared to no } \\
\text { history of diabetes) }\end{array}$ & $1.36(1.10-1.72)$ & 0.013 \\
\hline $\begin{array}{l}\text { Body mass index } \\
{\left[\mathrm{kg} / \mathrm{m}^{2}\right]}\end{array}$ & $0.98(0.97-0.99)$ & $<0.001$ \\
\hline $\begin{array}{l}\text { Geographic location: } \\
\text { rural }\end{array}$ & Reference & \\
\hline $\begin{array}{l}\text { Geographic location: } \\
\text { urban }\end{array}$ & $1.08(0.93-1.26)$ & 0.31 \\
\hline Hemoglobin $[\mathrm{g} / \mathrm{dl}]$ & $0.96(0.91-1.02)$ & 0.15 \\
\hline $\begin{array}{l}\text { Serum creatinine } \\
{[\mathrm{mg} / \mathrm{dl}]}\end{array}$ & $1.33(1.22-1.45)$ & $<0.001$ \\
\hline Serum albumin $[\mathrm{g} / \mathrm{dl}]$ & $0.44(0.33-0.58)$ & $<0.001$ \\
\hline $\begin{array}{l}\text { Serum cholesterol } \\
{[\mathrm{mg} / \mathrm{dll}]}\end{array}$ & $1.00(1.00-1.00)$ & 0.87 \\
\hline $\begin{array}{l}\text { Glycated hemoglobin } \\
\text { [\%] }\end{array}$ & $1.06(0.99-1.13)$ & 0.11 \\
\hline
\end{tabular}


Table IV. The SAI association with mortality in the entire population and study subgroup by proportional hazard model*

\begin{tabular}{|c|c|c|}
\hline & Hazard ratio $(95 \% \mathrm{Cl})$ & Value of $p$ \\
\hline $\begin{array}{l}\text { SAl in the entire } \\
\text { study population }\end{array}$ & $0.87(0.84-0.90)$ & $<0.001$ \\
\hline SAI in females & $0.91(0.86-0.97)$ & 0.005 \\
\hline SAl in males & $0.85(0.89-0.90)$ & $<0.001$ \\
\hline $\begin{array}{l}\text { SAl in non-Hispanic } \\
\text { black }\end{array}$ & $0.86(0.81-0.92)$ & $<0.001$ \\
\hline $\begin{array}{l}\text { SAl in non-Hispanic } \\
\text { white }\end{array}$ & $0.88(0.85-0.92)$ & $<0.001$ \\
\hline $\begin{array}{l}\text { SAl in Mexican- } \\
\text { American }\end{array}$ & $0.86(0.80-0.92)$ & $<0.001$ \\
\hline $\begin{array}{l}\text { SAl in urban } \\
\text { population }\end{array}$ & $0.88(0.83-0.93)$ & $<0.001$ \\
\hline $\begin{array}{l}\text { SAl in rural } \\
\text { population }\end{array}$ & $0.87(0.83-0.91)$ & $<0.001$ \\
\hline
\end{tabular}

${ }^{*}$ The data presented in the table were derived from five separate Cox models. Only hazard ratios for the SAl are indicated in the table. Each model was adjusted for age, sex, race, comorbidity index, history of diabetes, body mass index, geographic location (rural vs. urban), hemoglobin level, serum creatinine level, serum albumin level, serum cholesterol level, and glycated hemoglobin level

are clinically relevant. Despite a substantial effect size the discrimination ability of the model showed fairly modest results, where the C-index only changed from 0.62 to 0.64 by using SAl. However, it performed better than other established predictors of outcome such as comorbidity index, diabetes, and race; and it was comparable to age.

While this retrospective analysis was not designed to study the mechanism of the observed association, it is interesting to speculate about it. Based on our conceptual model, the subject could belong to a disadvantaged population simply due to either factors intrinsic to the subject (e.g., lack of motivation, limited developmental or intellectual resources, psychopathology, poor focus, low IQ [23]) or environmental factors (e.g., poor income of the parents, poor education opportunities) factors, or a combination of both factors. Resulting lower levels of social adaptability in turn might have social and healthcare implications.

We see several potential practical implications of the results. While an inferior outcome in underprivileged populations is evident, defining the population at risk based only on race, gender, and geographic location may be inadequate. Instead, our definition of an underprivileged population at risk for poor outcome is based on measuring the effect of belonging to this group (defined by lower SAI) and therefore should be more sensitive and specific. Indeed, based on our results, the increase in mortality associated with lower SAI values is substantial and represents high clinical significance.
The novelty and practicality of SAI probably deserves additional comments. The novelty of SAI is in its integrative nature and strong association with outcome. All of the individual components of SAl have been demonstrated to have an association with clinical outcome, including several reports by our group $[5,6,8,9,15]$. The effect size of each of these factors was relatively modest (less then 10\% risk reduction), while each point increase in SAI (the range is from 0 to 14) decreases the risk of death by $13 \%$. Furthermore, none of the individual factors comprising SAI can be used as a classifier in order to define an underprivileged population. On the other hand, SAI seems to be an excellent indicator of belonging to an underprivileged population at risk for healthcare disparities and adverse outcome. Researchers and practitioners may use SAI in designing interventions or clinical studies; it is easily measured and calculated; by implementing SAI the risk group can be defined more precisely, so that clinical interventions and resources may be focused and dispensed where they are needed the most.

This study has several limitations. As our analyses depend on the quality of reports from NHANES participants, potential misreporting and misclassification might be an issue, not only in this project, but also in other reports based on the same database. However, we chose variables that generally should be well reported and used categories which minimize participant reluctance to report actual income. As NHANES III enrolled a large number of participants, some statistically significant associations may be of limited clinical value. In this project however, we demonstrated a very high level of significance and high clinical relevance of the effect size.

Importantly, our results could be distorted by reverse causality described elsewhere [24]. In particular, one might imagine the situation where subjects with greater mortality probability might move towards underprivileged strata of the society with lower SAI. However, our results were robust after adjustment for comorbidity and a large number of health status indicators. In addition, the reader should realize that the NHANES study collection strategy was aimed at higher representation of African Americans and Hispanics than the general US population, so these populations in the dataset are over-represented. To address this issue we used weighted analysis, allowing data extrapolation to a larger randomly selected sample of the population.

In conclusion, we have developed an indicator of social adaptability that has a strong association with an individual's survival in the general US population and in various subgroups. This indicator can be used to identify underprivileged populations exposed to greater risk of death and target them for future interventions. 


\section{References}

1. Lederer DJ, Caplan-Shaw CE, O'Shea MK, et al. Racial and ethnic disparities in survival in lung transplant candidates with idiopathic pulmonary fibrosis. Am J Transplant 2006; 6: 398-403.

2. Mahle WT, Kanter KR, Vincent RN. Disparities in outcome for black patients after pediatric heart transplantation. J Pediatr 2005; 147: 739-43.

3. Keyhani S, Scobie JV, Hebert PL, McLaughlin MA. Gender disparities in blood pressure control and cardiovascular care in a national sample of ambulatory care visits. Hypertension 2008; 51: 1149-55.

4. Axelrod DA, Guidinger MK, Finlayson S, et al. Rates of solid-organ wait-listing, transplantation, and survival among residents of rural and urban areas. JAMA 2008; 299: 202-7.

5. Petersen E, Baird BC, Barenbaum LL, et al. The impact of employment status on recipient and renal allograft survival. Clin Transplant 2008; 22: 428-38.

6. Goldfarb-Rumyantzev AS, Koford JK, Baird BC, et al. Role of socioeconomic status in kidney transplant outcome. Clin J Am Soc Nephrol 2006; 1: 313-22.

7. Ikeda A, Iso H, Toyoshima $\mathrm{H}$, et al. Marital status and mortality among Japanese men and women: the Japan Collaborative Cohort Study. BMC Public Health 2007; 7: 73.

8. Naiman N, Baird BC, Isaacs RB, et al. Role of pre-transplant marital status in renal transplant outcome. Clin Transplant 2007; 21: 38-46.

9. Gueye AS, Chelamcharla M, Baird BC, et al. The association between recipient alcohol dependency and long-term graft and recipient survival. Nephrol Dial Transplant 2007; 22: 891-8.

10. Diaz VA Jr. Cultural factors in preventive care: Latinos. Prim Care 2002; 29: 503-17.

11. Gornick ME, Eggers PW, Reilly TW, et al. Effects of race and income on mortality and use of services among Medicare beneficiaries. N Engl J Med 1996; 335: 791-9.

12. Finkelstein JA, Lozano P, Farber HJ, Miroshnik I, Lieu TA. Underuse of controller medications among Medicaidinsured children with asthma. Arch Pediatr Adolesc Med 2002; 156: 562-7.

13. Gurwitz JH, Goldberg RJ, Malmgren JA, et al. Hospital transfer of patients with acute myocardial infarction: the effects of age, race, and insurance type. Am J Med 2002; 112: 528-34.

14. Priebe S, Watzke S, Hansson L, Burns T. Objective social outcomes index (SIX): a method to summarise objective indicators of social outcomes in mental health care. Acta Psychiatr Scand 2008; 118: 57-63.

15. Goldfarb-Rumyantzev AS, Rout P, Sandhu GS, Khattak M, Tang $H$, Barenbaum A. Association between social adaptability index and survival of patients with chronic kidney disease. Nephrol Dial Transplant 2010; 25: 3672-81.

16. Charlson ME, Pompei P, Ales KL, MacKenzie CR. A new method of classifying prognostic comorbidity in longitudinal studies: development and validation. J Chronic Dis 1987; 40: 373-83.

17. Pencina MJ, D'Agostino RB. Overall C as a measure of discrimination in survival analysis: model specific population value and confidence interval estimation. Stat Med 2004; 23: 2109-23.

18. Mussolino ME. Depression and hip fracture risk: the NHANES I epidemiologic follow-up study. Public Health Rep 2005; 120: 71-5.
19. Adamson SJ, Sellman JD, Frampton CM. Patient predictors of alcohol treatment outcome: a systematic review. J Subst Abuse Treat 2009; 36: 75-86.

20. Lissoni P, Messina G, Parolini D, et al. A spiritual approach in the treatment of cancer: relation between faith score and response to chemotherapy in advanced non-small cell lung cancer patients. In Vivo 2008; 22: 577-81.

21. Gerber Y, Goldbourt U, Drory Y. Interaction between income and education in predicting long-term survival after acute myocardial infarction. Eur J Cardiovasc Prev Rehabil 2008; 15: 526-32.

22. Wyke S, Ford G. Competing explanations for associations between marital status and health. Soc Sci Med 1992; 34: 523-32.

23. Batty GD, Shipley MJ, Gale CR, Mortensen LH, Deary IJ. Does IQ predict total and cardiovascular disease mortality as strongly as other risk factors? Comparison of effect estimates using the Vietnam Experience Study. Heart 2008; 94: 1541-4.

24. Greenberg JA. Hypothesis - the J-shaped follow-up relation between mortality risk and disease risk-factor is due to statistical confounding. Med Hypotheses 2002; 59: 568-76. 